\title{
Denudation type of transurethral resection for bladder tumor
}

\author{
Xiaofeng Yang*, Xiaojing Zhang, Hua Yang, Wei Zhang, Dongwen Wang \\ Department of Urology, First Affiliated Hospital of Shanxi Medical University, Taiyuan 030001, China
}

\begin{abstract}
Objectives: To investigate the safety and effectiveness of a denudation type of transurethral resection for bladder tumor (D-TURBT).

Methods: From December, 2000 to December, 2011, a total of 114 patients with nonmuscle invasive bladder cancer (NMIBC) were treated using D-TURBT by the same urologist for 121 person-times. The key steps of D-TURBT included removal of the visible tumors, electrocoagulation of the tumor basement membrane, and loop-electroexcision push-pull denudation of the coagulated tissues to expose the normal submucosa or muscle. The patients were followed up for $4.82 \pm 4.1$ years postoperatively to observe the efficacy and complications of D-TURBT.

Results: Among the 114 cases, 96 (84.21\%) had no recurrence after the first D-TURB, but 18 cases (15.79\%) relapsed. Those 18 patients underwent a second D-TURB; 13 (72.2\%) had no further relapse, but 5 cases had a second relapse. Those 5 patients underwent a third D-TURB; 2 of those cases had no further recurrence, but the other 3 had a third recurrence. Of those 3, 2 had distant metastasis, and 1 refused further treatment. During the follow-up, no patient experienced bladder perforation or was converted to open surgery. Only 2 $(1.75 \%)$ of the 114 cases showed worsened clinical staging, while 111 cases were tumor-free, for an overall response rate of $97.37 \%(111 / 114)$.

Conclusion: D-TURBT is an improved surgical approach. It removes NMIBC more effectively than conventional TURBT, while avoiding residual tumor and reducing the relapse rate. D-TURBT is an effective, safe, simple, and easy approach for treating NMIBC.

Keywords: Bladder tumor; Nonmuscle invasive bladder cancer; Denudation type of transurethral resection for bladder tumor; Recurrence
\end{abstract}

\section{INTRODUCTION}

Bladder cancer is a common malignant tumor of the urinary system. Overall, $75-85 \%$ of bladder tumors is confined to the mucosa (stage Ta or carcinoma in situ) or submucosa (stage T1) and is termed nonmuscle invasive or superficial bladder cancer. Nonmuscle invasive bladder cancer (NMIBC) is mainly treated by transurethral resection for bladder tumor (TURBT), which was first described by Stern and McCarthy in 1931. The TURBT approach has been continuously improved since then in the form of multiple surgical procedures, including standard TURBT ${ }^{[1]}$, repeated TURBT ${ }^{[2]}$, complete TURBT ${ }^{[3]}$, expanded TURBT ${ }^{[4]}$, and transurethral resection of whole bladder tumorall ${ }^{[5-}$ ${ }^{7]}$, all of which have aimed for complete removal of the tumor and reduction of tumor recurrence. However, the recurrence rates of solitary and multiple bladder tumors within 3 months remain at $0-15 \%$ and $7-45 \%$,

*Corresponding author: Xiaofeng Yang

Mailing address: Department of Urology, First Affiliated Hospital of Shanxi Medical University. Address: No.85 South Jiefang Road, Taiyuan 030001, Shanxi, China

E-mail: yxfylq@163.com

Fax: 0351-2143253

Received: 20 January 2018 Accepted: 20 March 2018 respectively ${ }^{[8,9]}$, indicating the urgent need for new surgical procedures.

The aim of the present study was to introduce a new surgical approach, the denudation type of transurethral resection for bladder tumor (D-TURBT), which we have developed based on our years of clinical experience.

\section{MATERIAIS AND METHODS}

Clinical data

From December, 2000 to December, 2011, a total of 114 patients, aged 45-85 years (mean, 63.15 \pm 18.23 years) were diagnosed with NMIBC according to the results of abdominal B ultrasound, cystoscopy and biopsy, and CT imaging. The preoperative clinical staging was based on CT images. The tumors were staged and graded by referencing the TNM staging and WHO grading systems ${ }^{[10,11]}$, All D-TURBT procedures (121 person-times) were performed by the same urologist.

Surgical procedure

All patients received epidural anesthesia and were placed in a lithotomy position, with routine disinfection and a sterile sheet. A $27 \mathrm{~F}$ continuous flow 

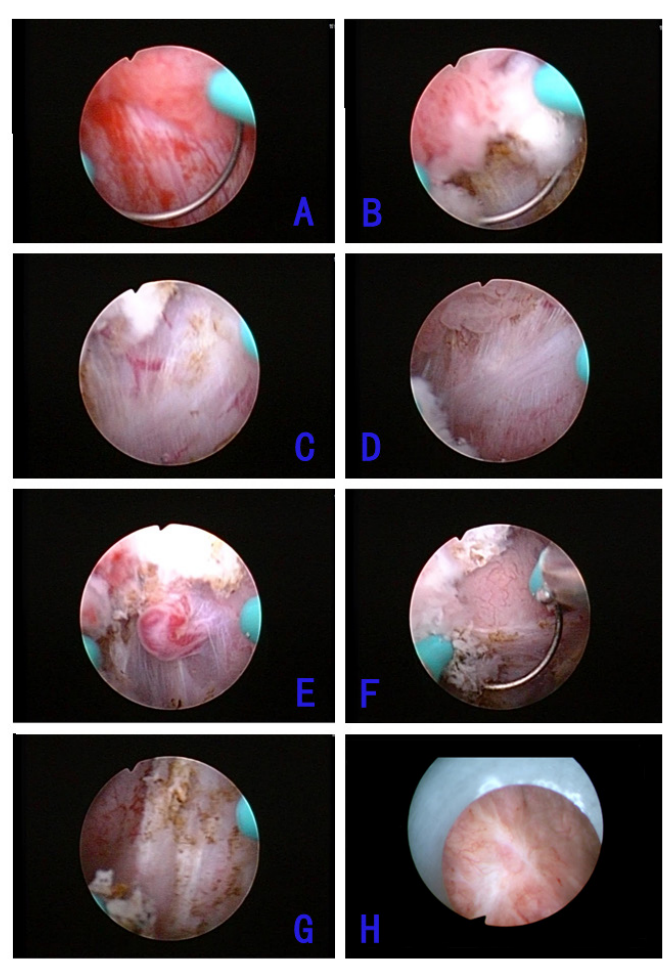

Figure 1. The key steps and skills of the TEUBT(A) The junction of normal bladder mucosa and bladder tumor; (B) Cutting the mucosa $0.5-1 \mathrm{~cm}$ outside from the junction; (C) Pushing the mucosa with diathermy loop until the tumor base; (D) Surface of the muscle; (E) The nutrient artery in the tumor pedicle; (F) Cut off the edge of tumor tissue and normal mucosa; (G) Normal structure of the base; $\mathrm{H}$ Healing of the tumor base after 3 months.

resectoscope (Olympus) was placed transurethrally, using a right-angle electroexcision loop and highfrequency medical electric knife. The cutting power and electrocoagulation power were $120 \mathrm{~W}$ and $60 \mathrm{~W}$, respectively, and the washing fluid was distilled water or $3 \%$ mannitol.

The key steps of D-TURBT were as follows: 1) removal of the visible tumor tissues to expose the tumor basement (Figure 1A and B); 2) electrocoagulation of the tumor basement into yellowish-black burned tissues (Figure 1C-F); 3) use of the electroexcision loop and the excision sheath to clamp the yellowish-black tissue at the junction between the normal mucosa and yellowish-black coagulated tissue, followed by pushing, pulling, etc., to denude the coagulated tissues from the bladder wall; repetition of the electrocoagulation and denudation for complete removal of the disorganized tumor tissue and other tissues with abnormal structure, until exposure of the normal lamina propria or muscle structure of the bladder wall (Figure 1G-I); 4) before finishing the surgery, repeated washing of the bladder with distilled water for complete removal of the floating tissues and eschar, until no floating solids and bubbles remain at the top inside bladder and the washing liquid becomes clear; placement of indwelling catheters and continuous rinsing with distilled water for 1 day; instillation of the bladder with mitomycin in the first day after surgery; and 5) histopathological examination (all patients underwent routine preoperative and postoperative tumor biopsy to determine the tumor grading).

\section{Follow up}

The bladder was treated with immediate intravesical instillation with mitomycin $(20 \mathrm{mg} / 40 \mathrm{~mL}$ saline $)$ within $24 \mathrm{~h}$ after the surgery. One week later, the intravesical mitomycin instillation was changed to once a week, for a total of 8 times, then once every 2 weeks for another 8 times, and once a month thereafter, for a total period of 1 year. After the surgery, cystoscopy was performed every 3 months. One year later, B-ultrasound was performed every 6 months for 3 years. Thereafter, B-ultrasound was performed annually. The 114 cases were followed up for 3 months to 11 years, with an average follow-up period of $4.68 \pm 4.1$ years.

\section{RESUITS}

Table 1. Clinical data.

\begin{tabular}{|c|c|c|c|c|c|c|}
\hline $\begin{array}{l}\text { Age } \\
\text { (Mean) }\end{array}$ & Sex Person-time(\%) & $\begin{array}{l}\text { Grade } \\
\text { Case (\%) }\end{array}$ & $\begin{array}{l}\text { Size } \\
\text { Case (\%) }\end{array}$ & $\begin{array}{l}\text { Number } \\
\text { Case (\%) }\end{array}$ & $\begin{array}{l}\text { Site } \\
\text { Case (\%) }\end{array}$ & $\begin{array}{l}\text { Time of } \\
\text { recurrence } \\
\text { Case }\end{array}$ \\
\hline \multirow{9}{*}{$\begin{array}{l}26-85 \\
\text { years } \\
(63.15 \\
\text { years })\end{array}$} & Male & Low & $<3 \mathrm{~cm}$ & Solitary & Parietal wall & $\leqq 3$ months \\
\hline & $103(85.2 \%)$ & $70(57.8 \%)$ & $93(76.9 \%)$ & $68(17.9 \%)$ & $7(5.7 \%)$ & 2 \\
\hline & Female & Medium & $3-6 \mathrm{~cm}$ & Multiple & Right wall & 3-6 months \\
\hline & $18(14.8 \%)$ & $18(14.8 \%)$ & $28(23.1 \%)$ & $31(82.1 \%)$ & $49(40.5 \%)$ & 4 \\
\hline & & High & $>6 \mathrm{~cm}$ & & Left wall & $6-9$ months \\
\hline & & $33(27.4 \%)$ & $0(0 \%)$ & & $\begin{array}{l}44(36.4 \%) \\
\text { Neck }\end{array}$ & $\begin{array}{l}5 \\
9-12 \text { months }\end{array}$ \\
\hline & & & & & $12(9.9 \%)$ & 7 \\
\hline & & & & & Trigone & $\geqq 12$ months \\
\hline & & & & & $9(7.5 \%)$ & 5 \\
\hline
\end{tabular}


Table 2. Results of D-TURBT

\begin{tabular}{llll}
\hline D-TURBT & $\begin{array}{l}\text { Case without/with } \\
\text { recurrence after 1st } \\
\text { D-TURBT }\end{array}$ & $\begin{array}{l}\text { Case without/with recurrence } \\
\text { after 2nd D-TURBT }\end{array}$ & $\begin{array}{l}\text { Case cured/with distal } \\
\text { metastasis after 3nd } \\
\text { D-TURBT }\end{array}$ \\
\hline First & $96 / 18$ & $13 / 5$ (1 refused further & \\
Second & & treatment) & $2 / 2$ \\
Third & & & \\
\hline
\end{tabular}

The blood glucose concentrations in diabetic rats after treatment with STZ solution were significantly higher than those in the control group ( $<<0.05)$ (Table 1).

\section{Disc degeneration in diabetic rats}

IIn this series of clinical data, the clinical staging was based on preoperative CT imaging, and the tumor was graded according to the preoperative cystoscopy biopsy. The size and number of tumors were based on CT images and intraoperative observation, as shown in Table 1 and 2. For this group of 114 patients with bladder cancer, the same urologist performed the D-TURBT, for a total of 121 person-times. After the first D-TURBT (114 surgeries/114 cases), 96 cases (84.21\%) showed disease-free survival within the follow-up period, while the other 18 cases (15.79\%) suffered a first relapse after a tumor-free period of 3-36 months (mean, 9.07 \pm 5.4 ). These 18 cases underwent a second D-TURBT; subsequently, 13 cases $(13 / 18,72.2 \%)$ had no further recurrence during the follow-up, but 5 cases relapsed again. Those 5 patients underwent a third D-TURBT, and 2 of them exhibited disease-free survival. Of these 2 patients, one was an 89-year-old male (as in 2012), who had been first diagnosed with bladder cancer in 2001. He received repeated transurethral electrocautery surgery, but this treatment failed to prevent relapse and the number of recurred tumors eventually increased. During his first D-TURBT in February, 2003, multiple tumors (high grade) were observed in the posterior and bilateral bladder walls, at various sizes $(1 \times 1.5 \mathrm{~cm}$ to $3 \times 3 \mathrm{~cm})$. Follow-up cystoscopy in July, 2003 revealed a recurred tumor (high grade) at the bladder neck with a diameter of about $1 \mathrm{~cm}$; this tumor was removed by a second D-TURBT. However, in October, 2005, follow-up again indicated a $1.5 \mathrm{~cm}$ lesion consisting of a red, round, moss-like mucosa in the parietal region inside bladder - a change resembling carcinoma in situand this lesion was then removed by third D-TURBT. The patient was followed up with cystoscopy annually thereafter and showed a tumor-free survival of 6 years as of the last follow-up.

Another patient was initially diagnosed with a $5-\mathrm{cm}$ bladder tumor in 1998 and underwent a partial cystectomy. In 2001, this patient was diagnosed with a $1-\mathrm{cm}$ tumor in the posterior urethral tract and underwent a first D-TURBT. Another tumor with a diameter of about $1 \mathrm{~cm}$ was found in the right posterior bladder wall and was removed by a second D-TURBT. The patient thereafter had a tumor-free survival during an 8-year follow-up.

In another 2 patients (2/114, 1.75\%), preoperative biopsy indicated a worsened tumor grading before the third D-TURBT, and intraoperative pathology showed that the infiltration of the tumor was too deep to resect and expose the normal tissue. A distant metastasis was detected shortly after the surgery. One of the 2 patients refused further therapy after the third relapse and later died from cardiovascular disease.

No bladder perforation or conversion to open surgery occurred in any patients during the follow-up. Only 2 patients showed worsened clinical staging and pathological grading $(2 / 114,1.75 \%)$. A total of 111 cases achieved tumor-free survival, resulting in a total effective rate of $97.37 \%(111 / 114)$.

\section{DISCUSSION}

Post-TURBT tumor recurrence has been an important challenge faced by urologists. In 1998, our group followed up 1845 patients with bladder cancer who had suffered recurrences, and found that, among them, 110 cases had 2-24 recurrences (mean, 3.09 times). The average tumor-free periods before the first, second, and third recurrences were 34.2, 18.1, and 11.6 months, respectively. The longest tumor-free survival was 10 years, and the recurrence declined gradually within 5 years after the surgery. The recurrence rates after 5-10 years became irregular, and the tumor staging rose after multiple recurrences. The 10-year survival rate was $56.73 \pm 6.7 \%{ }^{[12]}$.

As early as a century ago, Albarran and Imbert proposed that the cause of postoperative recurrence of bladder cancer was related to the seeding of floating cells, which could explain the up to $90.6 \%$ high recurrence rate in the parietal bladder wall ${ }^{[13]}$ Current molecular biological studies have now also confirmed the theory of a monoclonal origin for multiple 
tumor lesions. Adiyat et al. ${ }^{[3]}$ believed that $70 \%$ of the recurrences at the site of initial excision were caused by an incomplete tumor removal, and that the incomplete resection of tumors in the left wall was due to insufficient observation during the surgery; tumors in the anterior bladder wall were also difficult to resect, and tumors in the posterior and lateral bladder walls were also missed. Therefore, early recurrence was related to the presence of residual tumor cells, $30 \%$ of which were attributed to the residual carcinoma in situ.

TURBT is defined as a complete TURBT if the initial resection specimens contain the detrusor tissue, no residual tumor remains, and a second TURBT does not find residual tumor. The complete TURBT is an ideal surgical approach; however, except for including the detrusor tissue in the resection specimens, a reliable standard remains elusive for determining the complete resection of the tumor.

Daneshmand et al. ${ }^{[4]}$ proposed an expanded TURBT to overcome the problem of residual tumor post TURBT. In the expanded procedure, after a complete resection of the visible tumors, the resection area is expanded to include the center of tumor basement membrane, as well as the seemingly normal tumor edges seen under endoscopy. This approach is defined as an expanded TURBT if the resected tumor basement and surrounding tissues are diagnosed as negative by histopathological examination. The authors reported that the use of the expanded TURBT, which required an increased resection depth and area around the tumor basement, significantly decreased the rate of local recurrence to $5.1 \%$. However, we believe that this expanded resection represents a blind overtreatment. Other studies have suggested that TURBT needs to remove the tumor, but still comply with the "notumor" principle of surgical oncology. Many scholars have improved the electroexcision loop and proposed transurethral resection in one piece (TURBO) or en bloc resection ${ }^{[5-7]}$, to reduce the spread and seeding of tumor cells. However, in practice, not many tumors can be resected en bloc by TURBT, and most tumors larger than $1 \mathrm{~cm}$ require a gradual removal from the top to the basement. Therefore, complete removal of the tumor basement and clearing out of the floating tissues and cells inside the bladder are key technical issues that must be taken into account to avoid a second TURBT and prevent tumor cell seeding. However, a standard for a complete excision of the tumor basement is still lacking.

Some authors also showed that even if the detrusor tissues were observed in the resected tumor specimens, $4-29 \%$ of the tumor lesions were still not completely removed. In our experience, in actual surgery, we found that although most tumor lesions were graded as G1-2 and staged as Ta-T1, they had a wider basement, and not all tumors were pedunculated. Each cut involving detrusor tissue may cause bladder perforation and leakage of irrigation fluid, resulting in further spread and seeding of tumor cells outside the bladder. Therefore, these difficulties call for new techniques that can completely remove the tumor cells without causing bladder perforation.

The present series of operative approaches was designed in 2000, and the procedures were conducted by the same surgical urologists. The key aspect of D-TURBT is that, following the removal of the visible tumor tissues, the detrusor is not emphasized as a standard to determine the depth of the basement resection. Instead, electrocoagulation, electrosurgical excision, and simple mechanical pushing and pulling of the coagulated tissues are used to gradually expose the normal tissue or muscle. Especially when the range of the tumor basement is large, electrocoagulation can completely destroy the invisible tumor cells while still avoiding an excessively deep excision and the risk of bladder wall perforation. Collado et al. ${ }^{[14]}$ and Nieder et al. ${ }^{[15]}$ reported that bladderperforation is the most serious complication of TURBT, with an incidence rate as high as $1.3-3.5 \%$. In the present study, none of the 121 person-times of D-TURBT showed bladder perforation. We propose that the standard for a complete resection of the tumor by TURBT is the exposure of submucosa or muscular tissue that shows normal tissue structure, without damaging the muscle. Similarly, the sign of muscular invasion may serve as a criterion to determine whether a bladder cancer is NMIBC or at an advanced clinical staging. To achieve these resection standards, D-TURBT is a good procedure of choice.

The recurrence rate in this series of 114 patients after the first D-TURBT was $15.79 \%$. After the second D-TURBT, 13 recurrent cases were cured, but 5 recurred again and underwent a third D-TURBT. Thereafter, 2 of the 5 patients who had undergone the third D-TURBT suffered from distant metastasis, while another 2 cases were followed up without recurrence. Ultimately, except for 1 case who refused further treatment and 2 cases with distant metastasis and worsened clinical staging, all other patients (97.37\%) in this series showed no recurrence during the average $4.82 \pm 4.1$ years of follow-up.

Although 18 cases among this group of patients recurred after the first D-TURBT, their average followup time was $>5$ years, and intraoperative observations indicated that the recurrent lesions were not at the 
original resection site. These findings are significantly better than the results of Grimm et al., where 33\% of 124 cases that had undergone surgery displayed in situ tumor residues, and as many as $81 \%$ of their recurrences occurred at the site of the original lesion ${ }^{[16]}$. Our results are also superior to those of Kurth et al. ${ }^{[17]}$ and Zaharoff et al. ${ }^{[18]}$, who reported recurrence rates of up to $50-80 \%$ in a 1-year follow-up and 30 $50 \%$ in a 5 -year follow-up, respectively.

In the present study, the recurrence rate after the first D-TURBT was $15.79 \%$ in a 5 -year follow-up, and after third recurrence/D-TURBT, only $1.75 \%$ of the patients showed a worsened clinical staging. This was significantly lower than the traditionally held $10 \%$ of patients suffering from worsened clinical staging. In general, the mean follow-up time of our group of patients was $4.82 \pm 4.1$ years, during which 111 out of the 114 patients had tumor-free survival. Therefore, we believe that D-TURBT is a safe and effective surgical approach for treating NMIBC, with fewer complications and significantly reduced tumor recurrence in situ.

In conclusion, D-TURBT is an improved surgical approach over conventional TURBT. It shows certain efficacy in complete resection of the bladder tumor, avoidance of residual tumor, and effective reduction of the recurrence rate. It is an effective, safe, simple, and easy surgical approach for treating NMIBC, although an operation standard for TURBT remains to be established.

\section{ACKNOWLEDGEMENTS}

This study was supported by the fund from the National Natural Science Foundation of China (No. 81172444).

\section{REFERENCES}

1. Babjuk, M. (2009) Transurethral Resection of Non-muscle-invasive Bladder Cancer. European Urology Supplements 8, 542-548

2. Thomas, K., and O'Brien, T. (2008) Improving Transurethral Resection of Bladder Tumour: The Gold Standard for Diagnosis and Treatment of Bladder Tuwmours. European Urology Supplements 7, 524-528

3. Adiyat, K. T., Katkoori, D., Soloway, C. T., De los Santos, R., Manoharan, M., and Soloway, M. S. (2010) "Complete transurethral resection of bladder tumor": are the guidelines being followed? Urology 75, 365-367

4. Daneshmand, S. (2012) The value of extended transurethral resection of bladder tumour (TURBT) in the treatment of bladder cancer. BJU international 110, E80

5. Fallahi, M., Ubrig, B., and Roth, S. (2005) Transurethral en bloc resection of bladder tumours. Aktuelle Urologie 36, 31-32

6. Ukai, R., Hashimoto, K., Iwasa, T., and Nakayama, H. (2010) Transurethral resection in one piece (TURBO) is an accurate tool for pathological staging of bladder tumor. International journal of urology : official journal of the Japanese Urological Association 17, 708-714

7. Ganpule, A. (2012) En bloc transurethral resection of bladder lesions: a trick to retrieve specimens up to $4.5 \mathrm{~cm}$. BJU international 110, E158-159

8. Brausi, M., Collette, L., Kurth, K., van der Meijden, A. P., Oosterlinck, W., Witjes, J. A., Newling, D., Bouffioux, C., and Sylvester, R. J. (2002) Variability in the recurrence rate at first follow-up cystoscopy after TUR in stage Ta T1 transitional cell carcinoma of the bladder: a combined analysis of seven EORTC studies. European urology 41, 523-531

9. Babjuk, M., Oosterlinck, W., Sylvester, R., Kaasinen, E., Bohle, A., and Palou-Redorta, J. (2008) EAU guidelines on non-muscle-invasive urothelial carcinoma of the bladder. European urology 54, 303-314

10. Sobin, L. H. (2002) TNM classifi cation of malignant tumours. Journal of Clinical Pathology 51, 84

11. Epstein, J. I., Amin, M. B., Reuter, V. R., and Mostofi, F. K. (1998) The World Health Organization/ International Society of Urological Pathology consensus classification of urothelial (transitional cell) neoplasms of the urinary bladder. Bladder Consensus Conference Committee. The American journal of surgical pathology 22, 14 35-1448

12. Yang, X., Shen, P., and Kang, Q. (1998) Ten Year follow-up of recurrent bladder Cancer. Chin J Urol 19,530

13. Wilby, D., Thomas, K., Ray, E., Chappell, B., and O'Brien, T. (2009) Bladder cancer: new TUR techniques. World journal of urology 27, 309-312

14. Collado, A., Chechile, G. E., Salvador, J., and Vicente, J. (2000) Early complications of endoscopic treatment for superficial bladder tumors. The Journal of urology 164, 1529-1532

15. Nieder, A. M., Meinbach, D. S., Kim, S. S., and Soloway, M. S. (2005) Transurethral bladder tumor resection: intraoperative and postoperative complications in a residency setting. The Journal of urology 174, 2307-2309

16. Grimm, M. O., Steinhoff, C., Simon, X., Spiegelhalder, P., Ackermann, R., and Vogeli, T. A. (2003) Effect of routine repeat transurethral resection for superficial bladder cancer: a long-term observational study. The Journal of urology 170 , 


\section{3-437}

17. Kurth, K. H., Bouffioux, C., Sylvester, R., van der Meijden, A. P., Oosterlinck, W., and Brausi, M. (2000) Treatment of superficial bladder tumors: achievements and needs. The EORTC Genitourinary Group. European urology 37 Suppl $3,1-9$
18. Zaharoff, D. A., Hoffman, B. S., Hooper, H. B., Benjamin, C. J., Jr., Khurana, K. K., Hance, K. W., Rogers, C. J., Pinto, P. A., Schlom, J., and Greiner, J. W. (2009) Intravesical immunotherapy of superficial bladder cancer with chitosan/interleukin-12. Cancer research 69, 6192-6199 